\title{
Fertility preservation for patients with hematologic malignancies: The Korean Society for Fertility Preservation clinical guidelines
}

\author{
Dong-Yun Lee' ${ }^{1}$ Seul Ki Kim², Miran Kim³ ${ }^{3}$ Kyung Joo Hwang ${ }^{3}$, Seok Hyun Kim ${ }^{4}$ \\ 'Department of Obstetrics and Gynecology, Samsung Medical Center, Sungkyunkwan University School of Medicine, Seoul; ${ }^{2}$ Department of Obstetrics \\ and Gynecology, Seoul National University Bundang Hospital, Seongnam; ${ }^{3}$ Department of Obstetrics and Gynecology, Ajou University School of \\ Medicine, Suwon; ${ }^{4}$ Department of Obstetrics and Gynecology, Seoul National University College of Medicine, Seoul, Korea
}

Although the survival rate of hematologic malignancies in young patients is very high, cytotoxic therapies such as chemotherapy and total body irradiation therapy can significantly reduce a patient's reproductive capacity and cause irreversible infertility. Early ovarian failure also commonly occurs following additional cancer treatment, bone marrow transplantation, or autologous transplantation. Because the risk of early ovarian failure depends on the patient's circumstances, patients with a hematologic malignancy must consult health professionals regarding fertility preservation before undergoing treatments that can potentially damage their ovaries. While it is widely known that early menopause commonly occurs following breast cancer treatment, there is a lack of reliable study results regarding fertility preservation during hematologic malignancy treatment. Therefore, an in-depth discussion between patients and health professionals about the pros and cons of the various options for fertility preservation is necessary. In this study, we review germ cell toxicity, which occurs during the treatment of hematologic malignancies, and propose guidelines for fertility preservation in younger patients with hematologic malignancies.

Keywords: Fertility preservation; Hematologic malignancy

\section{Introduction}

The risk of infertility and early menopause due to ovulatory dysfunction caused by cancer treatment varies by the type of cancer and the treatment method used. Cytotoxic therapy, which is administered to premenopausal women with acute leukemia, Hodgkin lymphoma $(\mathrm{HL})$ and non-Hodgkin lymphoma (NHL), and chronic myeloid leukemia (CML), can induce infertility and early menopause. Hematopoietic cell transplantation (HCT) is also strongly associated with a risk of early menopause and infertility.

Received: Feb 28, 2017 · Revised: Mar 14, 2017 · Accepted: Mar 28, 2017 Corresponding author: Miran Kim

Department of Obstetrics and Gynecology, Ajou University School of Medicine, 206 World cup-ro, Yeongtong-gu, Suwon 16499, Korea

Tel: +82-31-219-5300 Fax:+82-31-219-5245 E-mail:kmr5300@ajou.ac.kr

This is an Open Access article distributed under the terms of the Creative Commons Attribution Non-Commercial License (http://creativecommons.org/licenses/by-nc/4.0/) which permits unrestricted non-commercial use, distribution, and reproduction in any medium, provided the original work is properly cited.
Acute myeloid leukemia (AML) and NHL usually develop at older ages. Patients younger than 50 comprise $20 \%$ of AML cases and $17 \%$ of $\mathrm{NHL}$ cases, and their cure rates are $50 \%$ to $60 \%$. However, the cure rates for $\mathrm{HL}(64 \%)$ and acute lymphoblastic leukemia (ALL, 75\%), which usually develop before the age of 50 , are $80 \%$ and $78 \%-92 \%$, respectively, which are considered to be very high [1]. More than half of all cases of ALL (56\%) occur among people aged less than 20 years. The remission rate of ALL has been reported to be $95 \%$ among teenagers and $78 \%-92 \%$ among adults [2].

While the survival rate of blood cancer that occurs at young ages is very high, chemotherapy and total body irradiation therapy performed as part of cancer treatment can significantly reduce a patient's reproductive capacity and cause irreversible infertility. In the present study, we review germ cell toxicity, which occurs during the treatment of hematologic malignancies, and propose guidelines for fertility preservation in younger patients with blood cancer. 


\section{Why does treatment of blood cancer lead to infertility?}

Typically, alkylating agents and radiation are used in blood cancer treatment, and they exhibit dose-dependent germ cell toxicity. The degree of gonadal damage during radiotherapy to the pelvic area, which includes the ovaries, is determined by the patient's age, the radiation dose, and the irradiation site. In general, a dose of $2 \mathrm{~Gy}$ can kill more than $50 \%$ of all ovarian follicles, and infertility and menopause can occur after high-dose irradiation of children and young women. Over $90 \%$ of women become menopausal after undergoing total body irradiation therapy prior to transplantation. According to the Childhood Cancer Survival Study, the pregnancy rate among cancer survivors is around $20 \%$ lower than among their siblings. The study also reported that the pregnancy rate decreased by $82 \%$ when women were exposed to $\geq 10 \mathrm{~Gy}$ [3]. Furthermore, the risk of infertility was three times higher for cancer survivors who underwent cancer treatment before the age of 20 [4], and the Bone Marrow Transplant (BMT) Survivor Study reported that the infertility rate was 36 times higher in transplant recipients [5].

The uterus can also be damaged by radiotherapy. Radiotherapy can increase the risk of spontaneous abortion, premature birth, and having a low-birth-weight baby. Women undergoing puberty, who are still in the process of maturation, are especially vulnerable to these risks.

For AML and ALL, the risk of gonadal damage due to radiotherapy alone is low as long as transplantation is not performed. The risk is also low with the use of tyrosine kinase inhibitors, which are used to treat CML. In the case of $\mathrm{HL}$, the risk of gonadal damage varies depending on the type of drug used. While doxorubicin, bleomycin, vinblastine, and dacarbazine (ABVD) used for early-stage cancer has a low risk, bleomycin, etoposide, adriamycin, cyclophosphamide, oncovin, procarbazine, and prednisone (BEACOPP) used for advanced or non-reactive cancer poses a high risk. Cyclophosphamide, doxorubicin, vincristine, and prednisone (CHOP) and cyclophosphamide, vincristine, dexamethasone, cytarabine, and methotrexate (CVAD) used for NHL pose a relatively low risk of gonadal damage. Transplantation is associated with a high risk of early ovarian failure, and total body irradiation therapy reduces ovarian function to a greater extent than does chemotherapy.

\section{How do patients with blood cancer differ from patients with other types of cancer?}

Since permanent amenorrhea has been reported to occur in less than $20 \%$ of all patients treated with the chemotherapeutic regimens that are typically used for $\mathrm{AML}, \mathrm{ALL}$, and $\mathrm{HL}$, fertility preserva- tion may appear to be unnecessary for most patients. However, in actuality, most cancer survivors have reduced ovarian function, experience subfertility, and have a shortened fertility window after chemotherapy [6].

Most patients diagnosed with acute leukemia and lymphoma must urgently undergo cytotoxic therapy and cannot afford to wait for 10 days of preparation before fertility preservation. Furthermore, because these patients are in a clinically unhealthy state due to cytopenia, the risks of intraoperative active infection, cardiopulmonary issues, or other possible complications must be considered.

Blood cancer has a high recurrence rate. Although permanent amenorrhea is rarely caused by the first round of cancer treatment, a patient may develop recurrent cancer after undergoing additional rounds of even more intense chemotherapy or HCT. During additional rounds of treatment, potent cytotoxic agents may be used or total body irradiation may be performed, and all of these lead to acute ovarian failure in most cases [7]. Therefore, it is important that patients seek health professionals' advice on future fertility even after their blood cancer treatment is completed.

CML mostly occurs after the age of 50 years, but $25 \%$ of cases occur among people aged 50 years or less. CML is treated with tyrosine kinase inhibitors (TKIs) over a long period of time, and this can affect fetal development and lead to infertility. TKIs increase the rate of natural abortion and fetal cardiac abnormalities [8-10]. Therefore, a patient who wishes to become pregnant in the future must consider discontinuing TKIs.

\section{Therapy}

\section{Hormone therapy}

Hormone therapy is currently the most controversial method of fertility preservation. According to a Cochrane meta-analysis that included results of multiple retrospective analyses, the use of gonadotropin-releasing hormone $(\mathrm{GnRH})$ analogs increased the rate of menstruation and of ovulation after cancer treatment 1.9-fold and 2.7fold, respectively, and significantly reduced the rate of amenorrhea. However, it ultimately did not affect the pregnancy rate or the number of antral follicles [11].

However, in studies on patients with lymphoma, GnRH agonist (GnRHa)-treated patients had a higher pregnancy rate than control patients [12], and regular ovulatory functions were likewise more significantly recovered in the GnRHa-treated group following autologous HCT [13]. However, these effects of GnRHa on ovarian preservation were not observed for patients with leukemia. Other studies have reported that GnRHa had no fertility-preserving effects $[14,15]$.

Recently, Demeestere et al. $[16,17]$ reported that after more than 5 years of follow-up, GnRHa administration did not significantly reduce 
primary ovarian failure or increase the pregnancy rate. Although several issues should be considered in interpreting the results of this study, GnRHa during chemotherapy remains an option for women interested in preserving ovarian function and fertility [18].

While there are reports that continuous use of combined oral contraceptives can lower the rate of amenorrhea and premature ovarian failure [19,20], combined oral contraceptives cannot be used as a primary method of fertility preservation since no prospective research comparing combined oral contraceptives-treated patients with those treated with other methods has been conducted.

Research on the effects of hormone therapy on the preservation of ovulatory functions is still lacking, and only a few studies on this topic have presented reliable results. Therefore, hormone therapy should be considered only when no other appropriate treatment options are available.

\section{Embryo or oocyte cryopreservation}

Embryo or oocyte cryopreservation is currently the most well-established method of fertility preservation $[21,22]$. Embryo or oocyte cryopreservation follows a similar procedure for blood cancers as for other types of cancer. With the recent introduction of the randomstart method, it has become possible to start stimulation at any time without having to wait for the next menstrual cycle, and it takes 10 to 14 days until oocyte retrieval. Random-start ovarian stimulation provides a significant advantage, and in emergent settings, can be started at a random cycle date for the purpose of fertility preservation without compromising oocyte yield or maturity [23]. The live birth rates among patients who attempted to become pregnant by thawing previously frozen oocytes and using them after completing cancer treatment were $35 \%$ to $40 \%$, which can be considered to be very high. Since oocyte cryopreservation as a method of fertility preservation is no longer in an experimental stage, it may become an option for active consideration by young female patients with cancer who currently have no partner [24].

However, many patients with blood cancer experience serious anxiety during the 10 days of stimulation before and after gamete cryopreservation or during the process of oocyte retrieval. They fear that their cancer treatment will be delayed due to gamete cryopreservation, and that they may develop complications as a result of the invasive procedure. However, if a patient is in a relatively healthy state, and has a sufficient desire to preserve her fertility, embryo or oocyte cryopreservation can be performed, as is done for patients with other types of cancer who desire fertility preservation.

\section{Ovarian tissue cryopreservation}

For patients with blood cancer, for whom gamete cryopreservation is difficult, ovarian tissue cryopreservation can be a useful method of fertility preservation. Ovarian tissue cryopreservation is superior to other therapies in several aspects. First, it can be performed right away without any waiting period and can be performed in the absence of a partner. Upon successful transplantation, hormone function returns to normal. Ovarian tissue cryopreservation can also be attempted for prepubertal children.

However, there is always a possibility of the presence of cancer cells in frozen ovarian tissue, and this possibility is higher for patients with blood cancer than for those with other types of cancer. While it has been reported that cancer cells do not invade the ovaries in most patients with lymphoma, this is not always true [25-28]. Despite this, autotransplantation of ovarian tissue has been frequently performed in patients with lymphoma, and so far, there has been no report of recurrent cancer following ovarian tissue cryopreservation and transplantation [25].

No case of autotransplantation of ovarian tissue in patients with leukemia has been reported to date. This is because in previous studies, cancer cells were detected $[24,28]$ during xenotransplantation of ovaries in patients with leukemia $[29,30]$ through a histological examination, polymerase chain reaction [31,32], or at autopsy [27]. Although some studies have reported not detecting any cancer cells after histological examination and xenotransplantation of ovaries of patients with leukemia [31,33], clinicians do not feel comfortable recommending autotransplantation of cryopreserved ovaries to patients with leukemia.

A group of fertility preservation specialists has recommended the use of the following methods to ensure the safety of patients with leukemia during ovarian tissue cryopreservation and transplantation. In vitro fertilization is not recommended for patients with AML or ALL who require urgent treatment. Ovarian tissue cryopreservation is also not recommended for these patients, since a large number of leukemic cells may be present in the bone marrow and blood during the acute phase.

AML treatment does not lead to serious loss of ovarian function [34]. However, patients who need to undergo an intense treatment that poses a high ovarian toxicity risk, such as high dose radiotherapy or bone marrow transplantation, in addition to the AML treatment should actively undergo fertility preserving treatment. Since cancer cells are not detected in the blood and bone marrow following the first round of cancer treatment in most patients, the probability of finding cancer cells in the ovaries is very low. However, the presence of residual leukemic cells in the ovaries must be checked using the most sensitive method possible prior to transplantation of the cryopreserved ovaries.

Because chemotherapy for ALL does not reduce ovarian function over a short period of time, a few rounds of chemotherapy can be performed to ensure the complete elimination of cancer cells in the 
blood before performing tissue cryopreservation. Because ALL mostly develops before the age of 20, oocyte retrieval from patients with ALL is difficult, and the effects of ovarian stimulation are reduced in these patients. Furthermore, since genetically abnormal oocytes retrieved after chemotherapy increase the rates of natural abortion and fetal abnormalities, in vitro fertilization, egg freezing, and in vitro maturation are not recommended for patients with ALL [35-37].

By performing ovarian tissue cryopreservation after administering chemotherapy and ensuring that no cancer cells are present in the blood and bone marrow, the additional risks associated with surgery can be avoided, and the possibility of reinvasion by cancer cells can be reduced.

Although fertility preserving treatment is generally not necessary for patients with $\mathrm{CML}$, these patients may consider undergoing ovarian tissue cryopreservation if TKI treatment fails to work or if transplantation becomes necessary due to a blast crisis. Ovarian tissue cryopreservation can also be performed for patients with HL before they complete two rounds of chemotherapy. Reinvasion by cancer cells is rare in these cases.

Taken together, cryopreservation of ovarian tissue is not a universally applicable option, but is an efficient option to preserve fertility in young patients facing or undergoing gonadotoxic therapies. It should be proposed only after careful evaluation of the surgical risks. Other options, such as vitrification of oocytes or embryos, may be a better choice for older patients or in patients with a high risk of neoplastic cells within the ovaries [38].

\section{Conclusion}

While early ovarian failure rarely occurs immediately after blood cancer treatment, early ovarian failure commonly occurs following additional cancer treatment, bone marrow transplantation, or autologous transplantation. Because the risk of early ovarian failure depends on the patient's circumstances, patients with blood cancer must consult health professionals regarding fertility preservation before undergoing treatments that can potentially damage their ovaries. While it is widely known that early menopause commonly occurs following breast cancer treatment, there is a lack of reliable study results regarding fertility preservation during blood cancer treatment. Therefore, an in-depth discussion between patients and health professionals about the pros and cons of the various options for fertility preservation is necessary.

\section{Conflict of interest}

No potential conflict of interest relevant to this article was reported.

\section{References}

1. Miller KD, Siegel RL, Lin CC, Mariotto AB, Kramer JL, Rowland JH, et al. Cancer treatment and survivorship statistics, 2016. CA Cancer J Clin 2016;66:271-89.

2. Inaba H, Greaves M, Mullighan CG. Acute lymphoblastic leukaemia. Lancet 2013;381:1943-55.

3. Green DM, Kawashima T, Stovall M, Leisenring W, Sklar CA, Mertens $A C$, et al. Fertility of female survivors of childhood cancer: a report from the childhood cancer survivor study. J Clin Oncol 2009;27:2677-85.

4. Barton SE, Najita JS, Ginsburg ES, Leisenring WM, Stovall M, Weathers RE, et al. Infertility, infertility treatment, and achievement of pregnancy in female survivors of childhood cancer: a report from the Childhood Cancer Survivor Study cohort. Lancet Oncol 2013;14:873-81.

5. Carter A, Robison LL, Francisco L, Smith D, Grant M, Baker KS, et al. Prevalence of conception and pregnancy outcomes after hematopoietic cell transplantation: report from the Bone Marrow Transplant Survivor Study. Bone Marrow Transplant 2006;37: 1023-9.

6. Rossi BV, Missmer S, Correia KF, Wadleigh M, Ginsburg ES. Ovarian reserve in women treated for acute lymphocytic leukemia or acute myeloid leukemia with chemotherapy, but not stem cell transplantation. ISRN Oncol 2012;2012:956190.

7. Assouline E, Crocchiolo R, Prebet T, Broussais F, Coso D, Gamerre $M$, et al. Impact of reduced-intensity conditioning allogeneic stem cell transplantation on women's fertility. Clin Lymphoma Myeloma Leuk 2013;13:704-10.

8. Abruzzese E, Trawinska MM, Perrotti AP, De Fabritiis P. Tyrosine kinase inhibitors and pregnancy. Mediterr J Hematol Infect Dis 2014;6:e2014028.

9. Ault P, Kantarjian H, O'Brien S, Faderl S, Beran M, Rios MB, et al. Pregnancy among patients with chronic myeloid leukemia treated with imatinib. J Clin Oncol 2006;24:1204-8.

10. Pye SM, Cortes J, Ault P, Hatfield A, Kantarjian H, Pilot R, et al. The effects of imatinib on pregnancy outcome. Blood 2008;111: 5505-8.

11. Chen H, Li J, Cui T, Hu L. Adjuvant gonadotropin-releasing hormone analogues for the prevention of chemotherapy induced premature ovarian failure in premenopausal women. Cochrane Database Syst Rev 2011;(11):CD008018.

12. Behringer K, Thielen I, Mueller H, Goergen H, Eibl AD, Rosenbrock J, et al. Fertility and gonadal function in female survivors after treatment of early unfavorable Hodgkin lymphoma $(\mathrm{HL})$ within the German Hodgkin Study Group HD14 trial. Ann Oncol 2012;23:1818-25. 
13. Blumenfeld Z, Patel B, Leiba R, Zuckerman T. Gonadotropin-releasing hormone agonist may minimize premature ovarian failure in young women undergoing autologous stem cell transplantation. Fertil Steril 2012;98:1266-70.e1.

14. Behringer K, Wildt L, Mueller H, Mattle V, Ganitis P, van den Hoonaard $B$, et al. No protection of the ovarian follicle pool with the use of $\mathrm{GnRH}$-analogues or oral contraceptives in young women treated with escalated BEACOPP for advanced-stage Hodgkin lymphoma: final results of a phase II trial from the German Hodgkin Study Group. Ann Oncol 2010;21:2052-60.

15. Demeestere I, Brice P, Peccatori FA, Kentos A, Gaillard I, Zachee P, et al. Gonadotropin-releasing hormone agonist for the prevention of chemotherapy-induced ovarian failure in patients with lymphoma: 1-year follow-up of a prospective randomized trial. J Clin Oncol 2013;31:903-9.

16. Demeestere I, Brice P, Peccatori FA, Kentos A, Dupuis J, Zachee P, et al. No evidence for the benefit of gonadotropin-releasing hormone agonist in preserving ovarian function and fertility in lymphoma survivors treated with chemotherapy: final long-term report of a prospective randomized trial. J Clin Oncol 2016;34: 2568-74.

17. Demeestere I, Brice P, Peccatori FA, Kentos A, Dupuis J, Zachee P, et al. Reply to M. Lambertini et al. J Clin Oncol 2017;35:805-6.

18. Lambertini M, Falcone T, Unger JM, Phillips KA, Del Mastro L, Moore HC. Debated role of ovarian protection with gonadotropin-releasing hormone agonists during chemotherapy for preservation of ovarian function and fertility in women with cancer. J Clin Oncol 2017;35:804-5.

19. Behringer K, Breuer K, Reineke T, May M, Nogova L, Klimm B, et al. Secondary amenorrhea after Hodgkin's lymphoma is influenced by age at treatment, stage of disease, chemotherapy regimen, and the use of oral contraceptives during therapy: a report from the German Hodgkin's Lymphoma Study Group. J Clin Oncol 2005;23:7555-64.

20. Blumenfeld Z, von Wolff M. GnRH-analogues and oral contraceptives for fertility preservation in women during chemotherapy. Hum Reprod Update 2008;14:543-52.

21. Practice Committee of American Society for Reproductive Medicine. Fertility preservation in patients undergoing gonadotoxic therapy or gonadectomy: a committee opinion. Fertil Steril 2013;100:1214-23.

22. Loren AW, Mangu PB, Beck LN, Brennan L, Magdalinski AJ, Partridge $\mathrm{AH}$, et al. Fertility preservation for patients with cancer: American Society of Clinical Oncology clinical practice guideline update. J Clin Oncol 2013;31:2500-10.

23. Cakmak H, Rosen MP. Random-start ovarian stimulation in patients with cancer. Curr Opin Obstet Gynecol 2015;27:215-21.
24. Practice Committees of American Society for Reproductive Medicine; Society for Assisted Reproductive Technology. Mature oocyte cryopreservation: a guideline. Fertil Steril 2013;99:37-43.

25. Bastings L, Beerendonk CC, Westphal JR, Massuger LF, Kaal SE, van Leeuwen FE, et al. Autotransplantation of cryopreserved ovarian tissue in cancer survivors and the risk of reintroducing malignancy: a systematic review. Hum Reprod Update 2013;19: 483-506.

26. Harris NL, Scully RE. Malignant lymphoma and granulocytic sarcoma of the uterus and vagina: a clinicopathologic analysis of 27 cases. Cancer 1984;53:2530-45.

27. Kyono K, Doshida M, Toya M, Sato Y, Akahira J, Sasano H. Potential indications for ovarian autotransplantation based on the analysis of 5,571 autopsy findings of females under the age of 40 in Japan. Fertil Steril 2010;93:2429-30.

28. Dolmans MM, Luyckx V, Donnez J, Andersen CY, Greve T. Risk of transferring malignant cells with transplanted frozen-thawed ovarian tissue. Fertil Steril 2013;99:1514-22.

29. Dolmans MM, Yuan WY, Camboni A, Torre A, Van Langendonckt A, Martinez-Madrid B, et al. Development of antral follicles after xenografting of isolated small human preantral follicles. Reprod Biomed Online 2008;16:705-11.

30. Turial S, Karabul N, Gutjahr P, Engel V, Bierschock S, Schier F. Ovarian tumours: late extramedullary recurrence of acute leukaemia. Eur J Pediatr Surg 2009;19:184-6.

31. Greve T, Clasen-Linde E, Andersen MT, Andersen MK, Sorensen SD, Rosendahl M, et al. Cryopreserved ovarian cortex from patients with leukemia in complete remission contains no apparent viable malignant cells. Blood 2012;120:4311-6.

32. Rosendahl M, Andersen MT, Ralfkiaer E, Kjeldsen L, Andersen MK, Andersen CY. Evidence of residual disease in cryopreserved ovarian cortex from female patients with leukemia. Fertil Steril 2010;94:2186-90.

33. Poirot CJ, Martelli H, Genestie C, Golmard JL, Valteau-Couanet D, Helardot $P$, et al. Feasibility of ovarian tissue cryopreservation for prepubertal females with cancer. Pediatr Blood Cancer 2007;49: 74-8.

34. Meirow D, Biederman $H$, Anderson RA, Wallace WH. Toxicity of chemotherapy and radiation on female reproduction. Clin Obstet Gynecol 2010;53:727-39.

35. Meirow D, Schiff E. Appraisal of chemotherapy effects on reproductive outcome according to animal studies and clinical data. J Natl Cancer Inst Monogr 2005;(34):21-5.

36. Kujjo LL, Chang EA, Pereira RJ, Dhar S, Marrero-Rosado B, Sengupta $S$, et al. Chemotherapy-induced late transgenerational effects in mice. PLoS One 2011;6:e17877.

37. Bar-Joseph H, Ben-Aharon I, Rizel S, Stemmer SM, Tzabari M, 
Shalgi R. Doxorubicin-induced apoptosis in germinal vesicle (GV) oocytes. Reprod Toxicol 2010;30:566-72.

38. Imbert R, Moffa F, Tsepelidis S, Simon P, Delbaere A, Devreker F, et al. Safety and usefulness of cryopreservation of ovarian tissue to preserve fertility: a 12-year retrospective analysis. Hum Reprod 2014;29:1931-40. 